\title{
Körper im/als Schaltkreis
}

DIY-Apparaturen und audiovisuelle Praktiken

sinnlicher Wahrnehmung

Robert Stock

\section{EINLEITUNG}

Seit einiger Zeit hat sich der Begriff body hacking zu einem neuen Schlagwort entwickelt. Es verweist einerseits auf Do-it-yourself(DIY)-Praktiken, die bis in die 1970er Jahre zurückzuverfolgen sind und im Bereich alternativer subkultureller Vergemeinschaftungen zu verorten sind. ${ }^{1} \mathrm{An}-$ dererseits beziehen sich body hacker auf das Hacker-Ethos und sind daran interessiert, auf Open-Access-Basis neue (digitale) Technologien zu entwickeln oder bereits vorhandene zu modifizieren, um somit weitere Nutzerpraktiken zu erschließen. In diesem Rahmen beabsichtigen sie, die Horizonte sinnlicher Wahrnehmungen zu verschieben oder diese möglicherweise gänzlich neu zu bestimmen. ${ }^{2}$ Dabei bringen die Vertreter der body hacking-Szene zu ihrer Selbstinszenierung verstärkt die Bezeichnung des Cyborgs ins Spiel, der in diesem Zusammenhang vor allem auf das Subkutanitätskriterium rekurriert. ${ }^{3}$ Dass diese Taktik als Performance im Rahmen einer durch Aufmerksamkeit strukturierten Öffentlichkeit zu verstehen ist, zeigt u.a. der Hinweis auf die Überlegungen von Amber

1 | Vgl. S. Duncombe: Notes from underground; A. Spencer: DIY; T. Triggs: Scissors and glue.

2 | Vgl. u.a. B. N. Duarte: Entangled agencies. Zu Hacking-Praktiken im Computer-Bereich und der Gentechnik vgl. S. Levy: Hackers; M. Wohlsen: Biopunk.

3 | Vgl. D. Spreen: Der Cyborg, S. 170. Grundlegend zum Begriff des Cyborgs vgl. D. Haraway: A manifesto for cyborgs. 
Case, die zu bedenken gibt, dass nicht nur implantierte Technologie, sondern schon der Gebrauch von Smartphones deren Nutzer als »Cyborgs« qualifizieren würde. ${ }^{4}$

An dieser Stelle sollen aber weniger die Praktiken der body hacker im Mittelpunkt stehen, wie sie von Tim Cannon oder anderen realisiert werden. ${ }^{5}$ Vielmehr fokussiert der vorliegende Beitrag den Zusammenhang von DIY-Apparaturen, menschlichen Körpern und künstlerischer Performance, um dabei Re-Konfigurierungen sinnlicher Wahrnehmungen, insbesondere jene des Hörens, aufzuzeigen. Ausgangspunkt ist zum einen die Überlegung, dass Praktiken der experimentellen Verschaltung des Körpers mit elektrischen Regelkreisen nicht unbedingt ein Novum der Digitalkultur darstellen. ${ }^{6}$ Zum anderen werden Körper und die Grenzen sinnlicher Wahrnehmung im Feld der Kunst zunehmend hinterfragt und ihre Bedeutungen zur Disposition gestellt.' Wie im Folgenden zu zeigen sein wird, sind Effekte dieser Prozesse ebenfalls im Bereich der DIY-Praktiken zu beobachten.

Der Hauptteil des Artikels analysiert die Werke von Neil Harbisson und Daito Manabe und beschreibt Konstellationen, die in Video-Aufzeichnungen von Performances aus dem Zusammenspiel verschiedener am Geschehen Partizipierender resultieren. Es geht insofern nicht um Performances als einmalige Ereignisse, sondern um deren Verfertigung im Rahmen audiovisueller Produktionen, die zwischen alternativer Netzkultur und kommerzialisierbaren Innovationen kontemporärer Populärkultur zu situieren sind. Ziel ist es, diejenigen Experimentalanordnungen und -räume nachzuvollziehen, die Körper als »dehnbare, sich dehnende und ausgedehnte, kulturell kodierte Gebilde« adressieren. ${ }^{8}$ Dabei gilt es zwar, die Relevanz technischer Objekte in der Performance-Kunst zu berücksichtigen. ${ }^{9}$ Auch die intrinsische Verknüpfung von Körpern und Praktiken wird bedacht. ${ }^{10}$ In einer weiterführenden Perspektive liegt der

4 | Vgl. A. Case: We are all cyborgs now.

5 | VgI. KPG: Bio-Hacking; M. Hoopenstedt: DIY-Cyborg.

6 | Vgl. A. Elsenaarr/R. Scha: Electric body manipulation as performance art; siehe auch den Beitrag von Bianca Westermann in diesem Band.

7 | Vgl. A. Jones: Body art; M. Schwartzman: See yourself sensing.

8 S. Schade: Hybride Ausdehnungen des Medialen und des Körpers, S. 62

9 | Vgl. C. Salter: Entangled, S. xxi-xxiii.

10 | Vgl. S. Hirschauer: Praktiken und ihre Körper.
Fokus in Anschluss an die Arbeiten von Knorr-Cetina und Rheinberger aber auf Experimentalräumen, in denen sich komplexe Prozesse der Wissensproduktion verorten. ${ }^{11}$ In der Konfiguration dieser Räume werden menschliche Körper, entsprechende techné und die Umgebung wechselseitig miteinander verfertigt. Dabei wird anhand der Fallbeispiele auch davon ausgegangen, dass menschliche Körper sich in genuiner Weise durch ihre Signalverarbeitungsstrategien als »electrical system « beschrei ben lassen und folglich als integrale Elemente der performierten Schaltkreise begriffen werden können. ${ }^{12}$ Insofern setzt sich der vorliegende Artikel von Argumenten der Enhancement oder Posthumanismus-Debatte $\mathrm{ab}$ - auch wenn diese von den Künstlern ins Gespräch gebracht werden, da dort prinzipiell von einer >Ganzheit< oder >Abgeschlossenheit< des Kör pers ausgegangen wird und der Fokus auf einer technisch-prothetischen Erweiterung bzw. Verbesserung liegt. ${ }^{13}$

\section{NeIL HARBisson: Eyeborg}

Vor rund zehn Jahren traf Neil Harbisson auf den Ingenieur Adam Montandon, zusammen entwickelten beide den Eyeborg. ${ }^{14}$ Es handelt sich um ein DIY-Implantat, das es Harbisson ermöglicht, Farben wahrzunehmen. Dieses Device operiert mit einer Kamera, verarbeitet Farbsignale, die im Sichtfeld des farbblinden Künstlers erscheinen, und gibt sie in Form von Audiosignalen aus. Während es zuerst an dessen Hinterkopf befestigt war und dort die Vibrationen auf den Schädelknochen übertrug und somit eine Wahrnehmung der Farben ermöglichte, hat sich Harbisson mittlerweile einen entsprechend mit dem elektronischen Auge verschalteten Sensor implantieren lassen. Durch die dauerhafte Verschaltung von Körper und dem Eyeborg verändert sich die Wahrnehmung des Künstlers und bedingt u.a., dass er im Traum keine Farben sehen, sondern sie vielmehr hören würde. In der Inszenierung seiner künstlerischen Identität nimmt

11 | Vgl. H-J. Rheinberger: Experimentalsysteme und epistemische Dinge; $\mathrm{K}$. Knorr-Cetina: Wissenskulturen.

12 A. Elsenaar/R. Scha: Electric body manipulation as performance art, S. 21.

13 | Vgl. kritisch dazu N. K. Hayles: How we became posthuman, S. xiv.

14 | Zu Montandon, der sich als "Experte für digitale Zukunft" bezeichnet vgl. http://www. adammontandon.com/ vom 13.03.2014 
Harbisson diesen Umstand als Anlass, um sich als »Cyborg « ${ }^{15}$ darzustellen. Es gehe ihm insofern auch nicht vorrangig um eine Kompensation seiner Sehbehinderung, als vielmehr darum, den Körper und die Sinne $\mathrm{zu} »$ hacken $\ll .{ }^{16}$

Entsprechend seiner Wahrnehmung, die er selbst als »Sonochromatismus « bezeichnet, tritt Harbisson auch in Szene: ${ }^{17}$ Er präsentiert sich mit Anzügen, Socken oder Schuhen, die - aus der Sicht Normalsehender - starke Farbakzente bieten und verweist mit diesem Auftreten auf die besondere Stellung der Art und Weise, wie er seine Umwelt und die darin vorkommenden Farben »sieht «. ${ }^{18}$

Um der veränderten Umweltwahrnehmung Harbissons nachzuspüren, an die ein ebenso modifiziertes Körperverständnis gebunden ist, wird nun auf einige kurze Videoclips eingegangen, die der Künstler auf Internetseiten und Video-Sharing-Plattformen veröffentlicht hat. Eine Variante, die Sicht- bzw. Hörweise des Eyeborgs filmisch zu erzeugen, kann anhand des Fruit Song nachvollzogen werden. Die Gestaltung des Videos ist sehr reduziert: Vor einem weißen Hintergrund erscheinen nacheinander verschiedene Früchte und Gemüse. Eine Ähnlichkeit dieser visuellen Abfolge mit der Kunst der Popart ist wohl nicht zufällig und unterstreicht, dass Harbisson sich in eine als innovativ geltende Kunst- und Musikproduktion einschreiben möchte. ${ }^{19}$ So zeigt das Video jede Frucht in Begleitung eines ständig sich wiederholenden kurzen Sinustons, der eben auf jener Frequenz angesiedelt ist, die der Eyeborg dem Blau der Pflaume, einer Orange, dem Gelb der Banane oder dem Dunkelblaugrün der Aubergine zuordnet. Das Obst wird je einzeln und nacheinander eingeblendet,

15 | Hier wird ein enger Cyborg-Begriff verwendet, der auf das Subkutanitätskriterium abhebt. Vgl. D. Spreen: Der Cyborg, S. 170.

16 | Vgl. TED Weekends. 'Hacking our senses،. WATCH: How a Colorblind Cyborg Hears Color. Siehe auch den Kommentar von E. Park: Ethical issues in cyborg technology.

17 | Vgl. C. F. Laferl/A. Tippner: Zwischen Authentizität und Inszenierung.

18 | Vgl. F. Falzeder: Body-Hacker und Cyborgs. Ein Mann hört rot.

19 | Das Cover der Debut-LP The Velvet Underground \& Nico (1967) gestaltete

Andy Warhol mit einer Banane auf weißem Hintergrund. Der Clou bestand darin, dass die Banane ein Aufkleberwar, hinter dem nach dem Abziehen eine rosafarbene Frucht erschien. Vgl. I. Ellis: Rebels wit attitude, S. 109. so dass in ihrer Abfolge eine melodische Tonfolge hörbar gemacht wird. ${ }^{20}$ Es handelt sich um eine Übersetzung der Wahrnehmung Harbissons, in der die Kamera die Perspektive des Eyeborgs einnimmt und dessen Lautproduktion auf der Tonspur eingespielt wird. Die Simulation trägt den Charakter eines Labor-Experiments, in der die Objekte rekonfigurier und damit störende Farbeinflüsse eliminiert werden, um so möglichst den reinen Klang einer einzelnen Frucht - wie er vom Eyeborg produziert wird - mess- und damit auch hörbar zu machen. ${ }^{21}$

Im Gegensatz zum Fruit Song setzen sich die sonochromatischen Porträts Harbissons dem vollen Farbspektrum eines Gesichts aus, in dem laut Auskunft des Künstlers 360 Farbnuancen im Spiel sind:

"Wenn ich das Gesicht von Menschen betrachte, höre ich unterschiedliche Töne Ich höre die Farbe inrer Haare, ihrer Augen, ihrer Lippen, ihrer Haut und schreibe die Noten auf eine Partitur. Von jedem Gesicht habe ich so einen Akkord. Es gibt hässliche Gesichter, die sehr harmonisch klingen[,] und schöne Gesichter, die sehr disharmonisch klingen. ${ }^{22}$

Eine filmische Herstellung der Wahrnehmung Harbissons bzw. des Eyeborgs bietet das sonochromatische Porträt des Fotografen Valentino Blas. ${ }^{23}$ Das Gesicht Blas ' wird in extremen Nahaufnahmen gezeigt, die in schwarz-weiß gefärbt sind und somit den farbblinden Blick Harbissons simulieren: zuerst das rechte Auge, dann das Haar, darauf folgt der Mund sowie das linke Auge und schließlich das Kinn. Eine Überblendung mit Unschärfe-Effekt leitet danach zur letzten Einstellung über, die das Gesicht im Ganzen zeigt. Die extremen Nahaufnahmen fragmentieren den Körper und schaffen die Voraussetzung, den Ton der Körperpartien zu bestimmen, aber selbst dies bleibt ambivalent, da jeweils mehrere Sinustöne im Spiel sind. Erst im Abspann werden die Messungen exakt angegeben und die genauen Frequenzen der beiden Augen, des Haares, der Haut und Lippen in der Form, wie sie der Eyeborg gemessen hat, einge-

20 | Vgl. N. Harbisson: Fruit Song

21 | Vgl. K. Knorr-Cetina: Wissenskulturen, S. 45-52; B. Latour: Gebt mir ein Laboratorium.

22 | J. Macher: Neil Harbisson - der Cyborg.

23 | Vgl. N. Harbisson: Sonochromatic Portrait. 
blendet. ${ }^{24}$ Zum Ende des Videos wird zudem deutlich, wie sich der Höreindruck des Gesichts bei Harbisson darstellt: Das Gemisch der Töne, die der Eyeborg produziert, und die hier auch einer weiteren soundtechnischen Bearbeitung unterzogen wurden, gerät zu einem anhaltenden Flimmern und Flirren. Der Blick der Kamera erzeugt in Kombination mit den Eyeborg-Sounds einen Eindruck derjenigen medialen Konfiguration, in der sich Harbissons Sehen und digital fabrizierte Klänge miteinander verschränken.

Eine andere Konkretion des künstlerischen visuell-vibrierenden Hörens findet sich in der Videoaufzeichnung eines Farbkonzerts, das im Mai 2013 in London stattfand. Dort kreierte Harbisson die Socken-Sonate Nr. 1, indem er sich unterschiedlich farbige Socken vor sein Gesicht und den Eyeborg hielt. ${ }^{25}$ Die so entstandenen Sounds unterzieht Harbisson im Verlauf der Performance einer Weiterverarbeitung auf seinem Laptop, wofür er digitale Audio- und Kompositionsprogramme verwendet. Die Eyeborg-Lautproduktion wird geloopt, mit Delays und anderen Effekten bearbeitet, so dass ein rhythmisiertes elektronisches Musikstück ertönt. Diese Komposition mag nicht besonders originell erscheinen. Sie erhält aber durch die Präsenz von Harbisson, der in gelber Hose und rotem Sacko die Bühne betritt, sowie durch die im Hintergrund an die Wand projizierten großformatigen Farbkuben - visuelle Effekte, die auf die Farbe der Socken verweisen - durchaus eine gewisse Unverwechselbarkeit. Im Rahmen dieser »apparativen Versuchsanordnung ${ }^{26}$ verschränken sich der Körper und die sinnliche Wahrnehmung Harbissons nicht mehr nur mit dem Eyeborg, sondern werden einem diffizilen Ensemble zugeordnet, das aus verschiedenen Agenturen besteht, die in ihrem Zusammenspiel die Wahrnehmungsweise des Eyeborgs performieren.

Harbisson betont in Interviews häufig, dass er den Eyeborg nicht nur für das konventionelle Farbspektrum, das dem menschlichen Sehen zugänglich ist, nutzen möchte. Er sei vielmehr daran interessiert, sowohl Infrarot- als auch ultraviolettes Licht wahrzunehmen:

24 | Das Auge wird z.B. mit einer Frequenz von $735.7263358 \mathrm{~Hz}$ angegeben. 25 | Vgl. Colour Concert. Neil Harbisson at TEDMEDLive Imperial College 2013. 26 | S. Schade: Hybride Ausdehnungen des Medialen und des Körpers, S. 62. "you can keep upgrading the senses and perceiving more and more the longeryou use it I guess. [...] I can now perceive near infrared and near ultraviolet, but the next stage is to perceive them from afar and just continue to extend this to be able to hear colours underwater and also in space. ${ }^{27}$

In dieser Beschreibung Harbisson klingen durchaus die Argumente bezüglich der Verbesserung des menschlichen Körpers an. ${ }^{28}$ Etwas das technisch machbar erscheint, sollte auch ausprobiert werden, argumentieren viele Vertreter aus den Reihen der sogenannten Bodyhacker oder Posthumanisten. ${ }^{29}$ Aus der Perspektive dieser Untersuchung erscheint es jedoch relevanter, dass - wenn der menschliche Körper wie eingangs beschrieben als elektrisches System begriffen und damit zum Bestandteil weiterer Schaltkreise werden kann - die offizielle Anerkennung seiner grundlegenden Technizität auf dem Spiel steht. So war Harbisson Mitbegründer der Cyborg Foundation und setzte sich dafür ein, dass die eng. lischen Behörden ein Passbild für seinen Ausweis akzeptierten, das ihn mit seinem Eyeborg zeigt. ${ }^{30}$ Diese Anerkennung sei ihm wichtig, gehöre das Device doch zu ihm, da es ihm nicht nur die Wahrnehmung von Farben erlaube, sondern integraler Teil seines Körpers sei: »I insisted that I wanted to have it included in the photo as it was an extension of my senses and a part of my body. $\ll^{31}$

\section{Verschaltungen im Split-Screen}

Das zweite Fallbeispiel setzt sich ebenfalls mit Praktiken der Verschaltung von Körpern und DIY-Apparaturen auseinander, wobei in diesem Fall die politische Dimension eine weniger große Rolle spielt, als das bei

27 | R. Bryant: People ,will start becoming technology،.

28 | Vgl. M. Christen: Der Einbau von Technik in das Gehirn, S. 197; siehe auch: C. Coenen (Hg.): Die Debatte über Human Enhancement،.

29 | Vgl. u.a. B. N. Duarte: Entangled agencies, S. 3.

30 | Vgl. Life with extra senses. Neil Harbisson and Moon Ribas at TEDxMuscat 2013 auf YouTube.

31 | Zitiert nach R. Bryant: People ,will start becoming technology،. Harbissons Schilderung ähnelt Kevin Warwicks Selbstdarstellungen und Experimenten. Vgl. K. Warwick: Cyborg morals, cyborg values, cyborg ethics, S. 134. 
Harbisson der Fall ist. Gerade auf der Ebene künstlerischer (Selbst-)Inszenierungen sowie in Bezug auf den Gebrauch audiovisueller Praktiken zur Adressierung sensorischer Wahrnehmungen bestehen jedoch gewisse Parallelen, wie gleich ersichtlich werden wird.

So wird etwa die Verschränkung körperlicher Bewegungen und apparativer Versuchsanordnungen auch in den Live-Performances des Trios Perfume aus Japan artikuliert. Daito Manabe, Programmierer und DIY-Experimentator, designte für die Tour der Girl-Band deren weiße Anzüge, die mit elektronischen Komponenten versehen sind und als Projektionsfläche für ein Kaleidoskop digitaler Graphik fungieren, wobei letztere zudem mit projizierten Hintergrundbildern auf der Bühne interagiert. Es handelt sich um »a combination of motion capture, visual programming and projection mapping technology ${ }^{32}$, die in ähnlicher Form auch von der Performance Eclipse/Blue entworfen wird..$^{33}$

Manabe und weitere seiner Mitstreiter der Gruppe Rhizomatiks widmen sich bereits seit einigen Jahren der Gestaltung experimenteller Umgebungen, die den menschlichen Körper und die sinnliche Wahrnehmung als zentralen Schauplatz des Geschehens bestimmen. ${ }^{34}$ Dies ist zum Beispiel im Video electric stimulus to face - test $3 \mathrm{zu}$ beobachten, das seit 2008 über eine Million Mal auf YouTube angeklickt wurde. ${ }^{35}$ Bei dem ca. dreiminütigen Clip handelt es sich um eine Webcam-Aufnahme, die nur aus einer Einstellung besteht. Das Gesicht Manabes, das mit einer Naheinstellung kadriert wird, erscheint in der Bildmitte und ist mit zahlreichen Elektroden versehen. Die Kabel führen aus dem Kader heraus, so dass die Art und Weise der Verschaltung nur erahnt werden kann. Wenn aber auf der Tonspur die abstrakte Elektro-Musik ertönt und das Künstler-Gesicht sich abrupte und rhythmisch beginnt zu bewegen, so wird klar, dass hier keine Körperdaten oder Gehirnströme gemessen werden. Vielmehr wird die Gesichtsmuskulatur Manabes als Zielobjekt elektrischer Impulse in Szene gesetzt, wobei unterschiedlichen Instrumenten bzw. Tonhöhen verschiedene Gesichtsregionen zugeordnet werden.

32 | J. Hadfield: Daito Manabe set to work his visual magic at Electraglide. Vgl. L. Jobey: Performance. Daito Manabe's light fantastic.

33 | Vgl. D. Manabe/N. Thing: Eclipse/Blue.

34 | Vgl. K. Holmes: Hacking the human body. Meet Daito Manabe; J. Noble: Daito Manabe.

35 | Vgl. D. Manabe: electric stimulus to face - test 3.
Die amateurhaft wirkende medizinische Versuchsanordnung stellt das DIY-Prinzip aus und verweist dabei - wenn auch in vereinfachter Form - auf die Anfänge elektronischer Musik mit ihren modularen Synthesizern und analogen Stecksystemen. ${ }^{36}$ Darüber hinaus werden im Rahmen der aufgezeichneten Performance auch Grenzen und Möglichkeiten des Hörens auf neue Weise verhandelbar. Indem das Gesicht durch die redu. zierte Kameraarbeit als Spektakel Kontur gewinnt, erhält die dort mit der Technik des »face visualizers « realisierte Choreographie auf der Basis der Wahrnehmung elektrischer Signale eine erhöhte Sichtbarkeit. ${ }^{37}$

In zugespitzter Form wird die per elektrischem Impuls induzierte Gesichtsstimulation von Manabe im Clip electricstimulus to face -test 7 einem Test unterzogen, wobei die Zahl der Probanden auf 36 Personen erhöht wird. ${ }^{38}$ Die vierminütige Aufzeichnung verwendet für die Darstellung des Tests einen Split Screen, so dass alle mit Elektroden und Kabeln versehenen Gesichter simultan im Kader erscheinen. Die Rekonfigurierung der Gesichter ist dabei nicht nur auf die Kadrierung durch Nahaufnahme zurückzuführen. In ähnlicher Weise wie die Objekte in Harbissons Fruit Song sind hier die Köpfe jeweils vor weißem Hintergrund platziert. Die Abkoppelung von einem subjektiven Raum bedeutet zugleich die Suspendierung anderer, in diesem Zusammenhang nicht relevanter körperlicher Regionen. Auf diese Weise wird die Zusammenführung der Gesichter in einem experimentellen Bildraum befördert. Auch räumliche Distanzen rücken in den Hintergrund und schaffen eine Beobachtbarkeit der verschiedenen Reaktionen auf elektrische Impulse. Zugleich erweist sich auch die zeitliche Ebene als relevant, denn die Gesichter, oder besser deren Bewegungen werden durch die Musik synchronisiert. Nicht einzelne Regungen oder individuelle Mimik erfahren eine Hervorhebung, sondern das als kollektiv konstruierte Wahrnehmungspotenzial der Gesichtsmuskeln. Die Strukturierung des Videos orientiert sich dabei an einer flachen Hierarchie, bei der die Musik zwar die zentrale Instanz darstellt, sie aber auf der auditiven Ebene angesiedelt ist und damit keine mittelbare Sichtbarkeit im Kader erhält, wie dies bei anderen Performances der Fall ist. ${ }^{39}$

36 | Vgl. T. Holmes: Electronic and experimental music, S. 240.

37 | Vgl. D. Manabe: Face visualizer. Face instrument.

38 | Vgl. D. Manabe: electric stimulus to face - test 7.

39 | Vgl. die Ausführungen zu Eric Whitacres "Virtual Choir" bei R. Reichert: Die Macht der Vielen, S. 142-144. 
Insofern operiert die Musik einerseits im Hintergrund. Andererseits wird sie durch Kabel und Elektroden gleichermaßen mit den Gesichtern auch visuell verbunden. So wird plausibel, dass das der Musik entsprechende akustische Frequenzspektrum über algorithmische Berechnungen die Körper der Teilnehmenden erreicht, dort gehört und in eine koordinierte Bewegungsabfolge der angesprochenen Muskeln übersetzt wird. Voraussetzungen dafür sind jedoch die Indifferenz und Reglosigkeit der Probanden gegenüber dem Reizfluss, dem sie sich aussetzen. Der Logik dieser »Partizipationsminimierung « ${ }^{40}$ widersetzen sich die Körper allerdings manchmal, wie vereinzelte Reaktionen der Probanden zeigen (wie z.B. Lachen oder schmerzhafte Gesichtsausdrücke).

In anderen Versuchsanordnungen ist es jedoch Manabe selbst, der sich als Dirigent fremder Muskelstrukturen ins Bild setzt. Dafür greift der japanische Experimentator auf myoelektrische Sensoren zurück, die u.a. zur Steuerung von Hand- oder Armprothesen entwickelt wurden. ${ }^{41}$ Diese Sensoren, die elektrische Spannungen von sich bewegenden Muskeln messen und in Steuersignale umwandeln, werden von Manabe zweckentfremdet. An der Hand des Musikers befestigt werden die ausgeführten Bewegungen genutzt, um einen Drum-Computer anzusteuern. Jedem Finger wird ein Schlaginstrument zugeordnet. ${ }^{42}$ In diese Operation wird eine weitere Person involviert: So zeigt das Testvideo Manabe zusammen mit einem Musikerkollegen, dessen Gesicht mit Elektroden versehen ist. Beide Probanden sind über Kabel mit einem weiteren, d.h. einem Computer verknüpft. Manabe improvisiert verschiedene Rhythmen, indem er mit den Fingern unterschiedliche Regionen des Gesichts der Testperson antippt. Der direkte Körperkontakt und die authentische Reaktion auf ihn werden in diesem Experimentalraum subvertiert: Denn die Gesichtsbewegung des Probanden ist nicht der Berührung zuzuschreiben, sondern dem elektrischen Steuerungsimpuls, der in Manabes Hand gemessen, über den Computer prozessiert, in Schlagzeugtöne übersetzt, dann an die Muskeln des Probanden zurückgeführt und schließlich als faziale Choreographie inszeniert wird. Der Clip, der aus nur zwei Einstellungen mit fester Kameraperspektive besteht, erreicht seinen Kulminationspunkt, als auch der Proband in das Geschehen eingreift: Indem er die Hand

40 | S. Hirschauer: Praktiken und inre Körper, S. 82.

41 |Vgl. u.a. C. Pylatiuk/L. Döderlein: Bionische Armprothesen, S. 1173.

42 Vgl. D. Manabe: electric stimulus to face-myoelectric sensor test 1.
Manabes berührt, setzt er sich selbst in die Lage, den eigenen Gesichtsausdruck mittels des elektrischen Schlagzeugspiels zu modellieren. ${ }^{43}$ Auf ähnliche Weise inszeniert auch der Versuchsaufbau im ersten Teil von Manabes Performance auf der Transmediale die künstlerisch gestaltbare Medialität von Individuationsprozessen. ${ }^{44}$ Dort wurde ein Kopierversuch wiederholt, der schon an anderer Stelle mit entsprechenden Ergebnissen vorgestellt wurde: Während Manabes expressive Mimik durch die in seinem Gesicht angebrachten myoelektronischen Sensoren gemessen wird, kann sie in abstrakte Geräusche übersetzt und zugleich auf die Gesichter seiner Musikerkollegen übertragen werden. ${ }^{45}$

\section{SCHLUSS}

Die Produktionen von Neil Harbisson und Daito Manabe entwerfen in exemplarischer Weise Verknüpfungen audiovisueller Praktiken und experimenteller Versuchsanordnungen, wobei erstere und letztere in einem Spannungsverhältnis zueinander stehen und sich wechselseitig verfertigen. So ordnet sich Harbissons Körper nicht nur dem Eyeborg, sondern einer umfassenden Apparatur zu, mittels derer sich sein »Sonochromatismus« konstituiert. Manabes Experimente sind auf die Einbeziehung mehrerer Körper angelegt, für welche sich Zumutungen und Herausforderungen hinsichtlich ihrer Teilhabe am »embodied act of musical performance ${ }^{46}$ stellen. In Anlehnung an Hauser kann hier von einer »biotechnological art« gesprochen werden, in der die Verschränkung sinnlicher Wahrnehmung mit DIY-Apparaturen erprobt wird. ${ }^{47}$ Obwohl in ihren Akzentuierungen verschieden verweisen Harbissons und Manabes Werke gleichermaßen auf den Bereich der Performance- und Video-Kunst, in der Experimente wie Stelarcs $»$ ping body $\aleph^{48}$ oder Arthur Elsenaars »remote « ${ }^{49}$ herkömmliche Konzeptualisierungen des Körpers

43 | Vgl. Ch. Salter: Entangled, S. 252

44 | Vgl. Daito Manabe. Performance at Transmediale 11. Berlin 2011.

45 | Vgl. D. Manabe: copy my facial expression into my friends' - test 0.

46 | Ch. Salter: Entangled, S. 217.

47 | Vgl. J. Hauser: Biotechnology as Mediality.

48 Vgl. V2 Institute for the Unstable Media: Stelarc.

49 | Vgl. A. Elsenaar: rEmote. a.k.a. Compose your emoticon. 
in Frage stellen. Solche Projekte setzen sich auf unkonventionelle Weise mit der Konzeptualisierung des menschlichen Körpers als elektrisches Signalsystem auseinander und transponieren dessen Bedeutungen durch seine Einbindung in weitere Schaltkreise.

Ob diese Praktiken dann aber tatsächlich Cyborgs bzw. »posthumane« Körper hervorbringen, ist sicher streitbar und zudem eine Frage des Labeling. Denn es ist nicht von der Hand zu weisen, dass die genannten Video-Performances in einer Populärkultur zu verorten sind, die sich zunehmend durch eine bionische Komponente zu charakterisieren scheint. Darauf lassen nicht nur die Videos und Auftritte von the first bionic pop star Viktoria Modesta oder die der Tänzerin Adrianne Haslet-Davis schließen. ${ }^{50}$ Auch das Projekt Mindtunes wäre zu nennen, in dem hippe Elektro-Sounds von Menschen mit körperlicher Behinderung per Elektroenzephalogramm sowie unter Mitwirkung von DJ Fresh kreiert wurde. ${ }^{51}$ Aber auch damit ist nur ein kleiner Ausschnitt eines wachsenden Felds umrissen, in dem Körper sowie künstlerische, experimentelle und audiovisuelle Praktiken aufeinandertreffen und das in Zukunft weiterer Analysen bedarf.

\section{VIDEOS}

》Colour Concert. Neil Harbisson at TEDMEDLive Imperial College 2013《, https://www.youtube.com/watch?v=6P8O5JXlAJg vom 13.03.2014.

»Daito Manabe. Performance at Transmediale 11. Berlin 2011«, https:// www.youtube.com/watch?v=nN5p4YgE2WM vom 13.12.2014.

Harbisson, Neil: »Fruit Song«, https://www.youtube.com/watch?v=HH2 voukljPs vom 13.03.2014.

Harbisson, Neil: »Sonochromatic Portrait. Valentino Blas, 2011«, https:// www.youtube.com/watch?v=KLyT-Oifywk vom 13.03.2014.

Manabe, Daito/ Nosaj Thing: »Eclipse/Blue«, https://www.youtube.com/ watch?v=QYsfrt188kA vom 05.12.2014.

Manabe, Daito: »copy my facial expression into my friends' - test o«, https://www.youtube.com/watch?v=VtRVvBSbonk vom 13.12.2014

50 | Vgl. L. Gannes: Dancing on a bionic leg; J. Plunkett: Pop riposte.

51 | Vgl. S. Kösch: Mental. Smirnoff Mindtunes.
Manabe, Daito: »electric stimulus to face - myoelectric sensor test $1 \ll$ https://www.youtube.com/watch?v=oh8YYONrLIc vom 13.12.2014.

Manabe, Daito: »electric stimulus to face - test $3 \ll$, https://www.youtube. com/watch?v=YxdlYFCp5Ic vom 13.12.2014.

Manabe, Daito: »electric stimulus to face - test 7«, https://www.youtube.

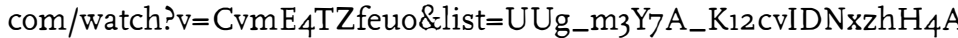
vom 13.12.2014

Manabe, Daito: »Face visualizer. Face instrument«, http://www.daito.ws/ en/work/smiles.html\#1 vom 13.12.2014.

\section{LITERATUR}

»Life with extra senses. Neil Harbisson and Moon Ribas at TEDxMuscat 2013《 vom 24.11.2013, https://www.youtube.com/watch?v=UEffjitNNM vom 13.03.2014

»TED Weekends. >Hacking our senses<. WATCH: How a Colorblind Cyborg >Hears< Color«, in: Huffington Post vom 25.07.2013, http://www.huffingtonpost.com/neil-harbisson/hearing-colorcyborg-tedtalk_b_3654445.html vom 05.11.2014.

Bryant, Ross: »People swill start becoming technology< says human cyborg«, in: dezeen magazine vom 20.11.201, http://www.dezeen. com/2013/11/20/interview-with-human-cyborg-neil-harbisson/ vom 05.11 .2014

Case, Amber: »We are all cyborgs now«, TED Talk, http://www.ted. com/talks/amber_case_we_are_all_cyborgs_now/transcript (13.05.2014).

Christen, Markus: »Der Einbau von Technik in das Gehirn. Das Wechselspiel von Informationsbegriffen und Technologieentwicklung am Beispiel des Hörens«, in: Barbara Orland (Hg.), Artifizielle Körper lebendige Technik technische Modellierungen des Körpers in historischer Perspektive, Zürich: Chronos 2005, S. 197-220.

Coenen, Christopher (Hg.): Die Debatte über >Human Enhancement Historische, philosophische und ethische Aspekte der technologischen Verbesserung des Menschen, Bielefeld: transcript 2010.

Duarte, Bárbara Nascimento: »Entangled agencies: New individual practices of human-technology hybridism through body hacking«, in: NanoEthics (2014): S. 1-11. 
Duncombe, Stephen: Notes from underground. Zines and the politics of alternative culture, London: Verso, 1997.

Ellis, Iain: Rebels wit attitude. Subversive rock humorists, Berkeley, CA: Soft Skull Press 2008.

Elsenaar, Arthur: »rEmote. a.k.a. Compose your emoticon«, in: artificial. Liberating the human face from tyranny of the brain, http://artifacial. org/remote vom 13.12.2014

Elsenaar, Arthur/Scha, Remko: »Electric body manipulation as performance art: A historical perspective«, in: Leonardo Music Journal 12 (2002): S. 17-28.

Falzeder, Florian: »Body-Hacker und Cyborgs. Ein Mann hört rot«, in: Tagesspiegel, vom 03.01.2014, http://www.tagesspiegel.de/medien/ digitale-welt/body-hacker-und-cyborgs-ein-mann-hoert-rot/9285758. html vom 04.11.2014.

Gannes, Liz: »Dancing on a bionic leg. The best Ted-Talk of $2014 \ll$, in: re/code vom 28.03.2014, http://recode.net/2014/03/28/dancing-on-abionic-leg-the-best-ted-talk-of-2014/ vom 15.12.2014.

Hadfield, James: »Daito Manabe set to work his visual magic at Electraglide«, in: The Japan Times vom 26.11.2013, http://www.japantimes. co.jp/culture/2013/11/26/music/daito-manabe-set-to-work-his-visualmagic-at-electraglide/\#.VN3nLPmG-VM vom 05.11.2014.

Haraway, Donna: $\gg$ A manifesto for cyborgs«, in: Socialist Review. 80 (1985), S. 65-108.

Hauser, Jens: $\gg$ Biotechnology as Mediality. Strategies of organic media art«, in: Performance Research 11.4 (2006), S. 129-136.

Hayles, N. Katherine: How we became posthuman. Virtual bodies in cybernetics, literature, and informatics, Chicago, Ill. u.a.: University of Chicago Press 1999.

Hirschauer, Stefan: »Praktiken und ihre Körper. Über materielle Partizipanden des Tuns«, in: Karl Hörning/Julia Reuter (Hg.), Doing Culture: Neue Positionen zum Verhältnis von Kultur und sozialer Praxis, Bielefeld: transcript 2004, S. 73-91.

Holmes, Kevin:»Hackingthehumanbody.MeetDaito Manabe«, in:TheCreators Project vom 03.01.2013, http://thecreatorsproject.vice.com/blog/ hacking-the-human-body-meet-daito-manabe vom 05.11.2014

Holmes, Thom: Electronic and experimental music. Technology, music and culture. London: Routledge 2012.
Hoopenstedt, Max: »DIY-Cyborg《, in: Motherboard vom 31.10.2013, http:// motherboard.vice.com/blog/the-diy-cyborg vom 15.12.2014.

Jones, Amelia: Body art. Performing the subject, Minneapolis u.a.: University of Minnesota Press 1998.

Knorr-Cetina, Karin: Wissenskulturen. Ein Vergleich naturwissenschaftlicher Wissensformen, Frankfurt a.M.: Suhrkamp 2002.

Kösch, Sascha:»Mental. Smirnoff Mindtunes«, in: debug vom 12.06.2013, http://de-bug.de/musik/mental-smirnoff-mindtunes/vom 15.12.2014.

KPG: »Bio-Hacking: Amerikaner lässt sich Kopfhörer implantieren«, in: Spiegel Online vom 03.07.3013, http://www.spiegel.de/netzwelt/ gadgets/bio-hacking-richard-lee-implantiert-sich-magneten-fuertonuebertragung-a-909154.html vom 13.03.2014.

Laferl, Christopher F./Tippner, Anja: »Zwischen Authentizität und Inszenierung. Künstlerische Selbstdarstellung im 20. und 21. Jahrhundert«, in: Dies. (Hg.), Künstlerinszenierungen. Performatives Selbst und biographische Narration im 20. und 21. Jahrhundert, Bielefeld: transcript 2014, S. 15-36.

Latour, Bruno: „Gebt mir ein Laboratorium und ich werde die Welt aus den Angeln heben«, in: Andrea Belliger/David J. Krieger (Hg.), ANThology. Ein einführendes Handbuch zur Akteur-Netzwerk-Theorie, Bielefeld: transcript 2006, S. 103-134.

Levy, Stephen: Hackers. Heroes of the computer revolution, Garden City, N. Y.: Anchor Press 1984.

Macher, Julia: »Neil Harbisson - der Cyborg. Farbenblinder Künstler übersetzt mit einem elektronischen Auge Farben in Töne und Töne in Farben«, in: Deutschlandradio Kultur vom 20.01.2011, http:// www.deutschlandradiokultur.de/neil-harbisson-der-cyborg.1153.de. html?dram:article_id $=182394$ vom 04.11.2014.

Noble, Joshua: »Daito Manabe. being real about being material«, in http://www.creativeapplications.net/theory/daito-manabe-being. real-about-being-material-theory/vom 22.05.2011.

Otsuki, Grant Jun: »Augmenting Japan's Bodies and Futures: The Politics of Human-Technology Encounters in Japanese Idol Pop«, in: M/C Journal 16.6 (2013): http://www.journal.media-culture.org.au/index. $\mathrm{php} / \mathrm{mcjournal} /$ article/view/738 vom 05.11.2014.

Park, Enno: »Ethical issues in cyborg technology. Diversity and inclusion«, in: NanoEthics (2014), S. 1-4. 
Plunkett, John: »Pop riposte. Channel 4 invades X Factor ad 'break«, in: The Guardian vom 11.12.2014, http://www.theguardian.com/ media/2014/dec/11/channel-4-x-factor-viktoria-modesta-prosthetic-riposte vom 15.12.2014.

Pylatiuk, C./Döderlein, L.: »Bionische Armprothesen. Stand der Forschung und Entwicklung«, in: Der Orthopäde 35 (2006), S. 1169-1175.

Reichert, Ramón: Die Macht der Vielen. Der neue Kult der digitalen Vernetzung. Bielefeld: transcript 2013

Salter, Chris: Entangled. Technology and the transformation of performance, Cambridge, Mass. u.a.: MIT Press 2010.

Schade, Sigrid: »Hybride Ausdehnungen des Medialen und des Körpers. Eine kleine Geschichte des Körperbildes in Medien/Kunst $\ll$, in: Claudia Pantellini (Hg.), Body extensions art, photography, film, comic, fashion, Stuttgart: Arnoldsche 2004, S. 56-71.

Schwartzman, Madeline: See yourself sensing. Redefining human perception, London: Black Dog Publishing 2011.

Spencer, Amy: DIY. The rise of lo-fi culture, New York: Marion Boyars, 2005.

Spreen, Dirk: »Der Cyborg. Diskurse zwischen Körper und Technik«, in: Eva Esslinger (Hg.), Die Figur des Dritten ein kulturwissenschaftliches Paradigma, Frankfurt am Main; Berlin: Suhrkamp 2010, S. 166179.

Triggs, Teal: »Scissors and glue. Punk fanzines and the creation of a DIY aesthetic«, in: Journal of Design History 19.1 (2006), S. 69-83.

V2_ Institute for the Unstable Media: »Stelarc«, http://v2.nl/archive/ people/stelarc/ vom 13.12.2014.

Wohlsen, Marcus: Biopunk. DIY scientists hack the software of life. New York: Current, 2011.

Zylinska, Joanna (Hg.): The Cyborg experiments. The extensions of the body in the media age, London/New York: Continuum 2002. 